\title{
The Effects of Magnetic Fields on the Structure of Cholesteric Liquid Crystals of Polypeptides
}

\author{
Eisaku IızukA \\ Faculty of Textile Science and Technology, \\ Shinshu University, Ueda, Japan.
}

(Received July 24, 1972)

\begin{abstract}
The change of pitch for the helical structure of cholesteric liquidcrystalline solutions of poly( $\gamma$-benzyl-L-glutamate) and of poly( $\gamma$-ethyl-L-glutamate) in static magnetic fields has been observed by means of diffraction of light from a laser. Both solutions sense the influence of magnetic fields though not so strongly in the latter case as in the former case. Values of the elastic moduli, $k_{22}$ and $k_{33}$, characterizing the structure have been evaluated. The value of $k_{22} /\left(\chi_{\mathrm{p}}-\chi_{\mathrm{t}}\right)$ ranges from 1 to $10, k_{33}$ being almost equal to, or slightly smaller in magnitude than $k_{22}$. The magnetic anisotropy, $\chi_{\mathrm{p}}-\chi_{\mathrm{t}}$, would seem to decrease when the polymer concentration is decreased.

KEY WORDS Liquid Crystal / Cholesteric Structure / Polypeptide / Magnetic Susceptibility / Electric Field / Diffraction of Light / Polarizing Micrograph /
\end{abstract}

Robinson, ${ }^{1}$ in explaining his optical observations on liquid-crystalline solutions of $\operatorname{poly}(\gamma-$ benzyl-L-glutamate) (PBLG), has put forward a cholesteric model in which there are a number of layers of approximately molecular thickness, with the molecules in each layer having a preferential orientation within the layer, and the direction of this orientation being rotated through a small, constant angle (about 2 or $3 \mathrm{~min}$ ), always in the same direction as one passes from one layer to the next. Each molecule, however, does not maintain a precise orientation because of brownian movement, though not enough disorientation occurs to break up the cholesteric structure. Visible equidistant parallel lines, associated with an oscilating value of retardation, are shown by the cholesteric solution when observed with light parallel to the layers of molecular alignment. The very large periodicities in the ability to pass light through solution makes it possible to obtain diffraction patterns with visible light.

Electric or magnetic fields acting on the anisotropy of the electric or magnetic susceptibility exert torques within a liquid crystal which may compete with the elastic torques determining its internal structure. From this point of view, Mayer ${ }^{2}$ has proposed a few experiments and has derived equations which are easily tested on the basis of Frank's theory ${ }^{3}$ of curvature-elasticity in molecularly uniaxial liquid crystals. In doing this, he has determined the structure which minimizes the total free energy, subject to boundary conditions concerning only four independent elastic moduli, $k_{11}, k_{22}, k_{33}$, and $k_{24}$, and the anisotropy of the magnetic susceptibility, $\chi_{\mathrm{p}}-\chi_{\mathrm{t}}$.

Since Sobajima's finding ${ }^{4}$ on the magnetic orientation of liquid crystals of PBLG, quite a few works on this problem have been presented. ${ }^{5-11}$ Yomosa, ${ }^{12}$ in explaining the mechanism for the magnetic orientation of PBLG, has proposed the idea that very large paramagnetic effects are produced by charge transfer with inpurity oxygen atoms leading to holes in the benzene rings of the side-chain groups. While Suzuki and his coworkers ${ }^{13}$ have calculated interaction energies between a constant magnetic field and valence shell electrons for peptide groups of the main chain, and have evaluated the diamagnetic and paramagnetic terms as the order of $10^{-8} \mathrm{eV}$ at 14 kilogauss. This suggests that all the polypeptide molecules are able to undergo magnetic orientation when the conditions are suitable.

Quite recently, the author ${ }^{14}$ reported that 


\section{E. IIZUKA}

concentrated solutions of PBLG oriented very well in the direction of a very low electric field such as $84 \mathrm{~V} / \mathrm{cm}$. This has been well explained by assuming the presence of independent molecular clusters of PBLG with dipole moments some 730 times as large as that of the single polymer (of degree of polymerization, 650). This is especially true in dilute liquid-crystalline solutions whose concentrations, however, are beyond Robinson's critical concentration, B (see ref 15). ${ }^{16}$ The starting solution, in a quartz cell of $1.00-\mathrm{mm}$ thickness with a $0.90-\mathrm{mm}$ quartz spacer, is not cholesteric. Sufficient conditions to believe that rodlike molecular clusters are easily produced in a low electric field has been obtained from the scattering of laser light. ${ }^{17}$ The critical field strength to produce the molecular clusters is dependent on the polymer concentration. The magnetic orientation has been elucidated by assuming the presence of molecular clusters. ${ }^{11}$

So far, the only test specimen that is reported to orient in magnetic fields, is limited to PBLG. Even PBLG has not been observed to undergo the magnetic orientation when dioxane is used as a liquid-crystalline solvent, however there is no explanation for this. ${ }^{*}$ The cholesteric structure is a kind of continuous body and is considered to be very susceptible to external fields. The purpose of this paper is to present results concerning effects of magnetic fields on the cholesteric structure of liquid-crystalline solutions of PBLG as compared with that of PELG [poly( $\gamma$-ethyl-L-glutamate)]. Values of some physical measurements which bear on determining the structure will be evaluated.

\section{EXPERIMENTAL}

The PBLG used was of mean degree of polymerization 1100 and was prepared from the $N$-carboxy- $\alpha$-amino acid anhydride of $\gamma$-benzylL-glutamate by Prof. T. Hayakawa of this Faculty. The PELG used was supplied by Ajinomoto Co., Ltd., as an ethylacetate solution with an intrinsic

* Sobajima has put forward the idea that PBLG is able to orient even in dioxane based on his NMR observation ${ }^{4}$ and has sufficient evidence that poly $(\gamma$-methyl-L-glutamate) undergoes the magnetic orientation. ${ }^{18}$ viscosity 1.42 in dichloroacetic acid at $30^{\circ} \mathrm{C}$, and was used after being dried. Each polymer was dissolved in a solvent such as dioxane or $\mathrm{CH}_{2} \mathrm{Cl}_{2}$ that allows the formation of cholesteric liquid crystal (and of $\alpha$-helices) and kept 2--3 weeks in a sample tube at room temperature. The solution was then put in a rectangular quartz cell of path length $1 \mathrm{~mm}$.

The cell with the specimen inside was placed, with its surfaces parallel to the magnetic field, between the poles of a permanent magnet made by Mitsubishi Steelworks Co., Ltd. The field strength was changed in a range between 6.0 and 9.6 kilogauss by adjusting the gap length of the magnet. A He- Ne gas laser model 132J was supplied by Japan Laser Co., Inc. and produced a 1-mW light with a wavelength of $6328 \AA$. Using this laser, light diffraction patterns of the cholesteric solution were projected onto a ground glass plate and photographed with a camera using $35-\mathrm{mm}$ film. The diffraction patterns were also photographed in static electric fields supplied by dry cells through a couple of $0.95-\mathrm{mm}$ thick platinum needle electrodes placed $0.75-\mathrm{mm}$ apart inside of cell. The direction of the external field was horizontal and portions of the solution were chosen where the field direction was parallel or perpendicular to the molecular layers of the cholesteric structure. The exposure time was $1 / 15 \mathrm{sec}$ at $\mathrm{f}: 3.5$ when the distance between the specimen and the glass plate was $50 \mathrm{~cm}$ and the film sensitivity was DIN : 24 .

The temperature of the specimen was $22^{\circ} \mathrm{C}$ unless stated otherwise and regulated by circulating water around the core of the magnet. The solvents used were of reagent grade and were used without further purification. All the solutions used were composed only of the birefringent phase and the values of the polymer concentration were very approximate.

\section{RESULTS AND DISCUSSION}

When observed with a low-power micrograph, the visible periodicity appeared after 1 to several days depending on the system being observed. The diffraction patterns were obtained with visible light. Using the equation for a diffraction grating, $m \lambda=d \sin \theta$, where $\theta$ is the half-angle 
The Effects of Magnetic Fields on the Structure of Cholesteric Liquid Crystals of Polypeptides
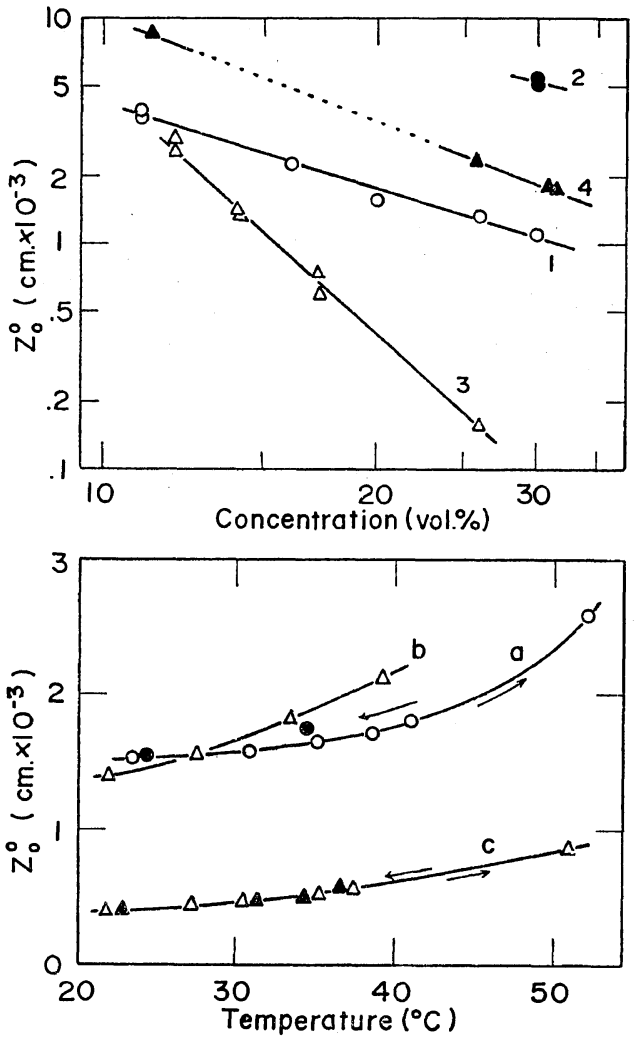

Figure 1. Double logarithmic plot of $Z_{0}{ }^{0}$ against concentration (upper) and $Z_{0}^{0} v s$. temperature relationship (lower): 1, PBLG/dioxane; 2, PBLG/ $\mathrm{CH}_{2} \mathrm{Cl}_{2} ; 3$, PELG/dioxane; 4, PELG/ $\mathrm{CH}_{2} \mathrm{Cl}_{2}$. a, PELG in dioxane (16.1 vol \%); b, PELG in dioxane (14.0 vol \%); c, PELG in dioxane (17.3 vol \%). subtended by the diffraction spots, the distance $d$ between the lines in the grating was determined to be in close agreement with the visible periodic spacing. The angle the diffracted ray makes with the incident beams within the solution, is different from that in air. ${ }^{19}$ However, the wavelength of the beam in the solution is $\lambda / n$, where $n$ is the refractive index of the solution, and the above situation needs not to be taken into consideration as far as this equation is concerned. The pitch of the uniform helical structure was determined as $Z_{0}{ }^{0}=2 d$. It is dependent on the polymer concentration as well as on temperature as may be seen in Figure 1. The results for PBLG in dioxane and PELG in $\mathrm{CH}_{2} \mathrm{Cl}_{2}$ show that $Z_{0}^{0}$ is proportional to $1 / C^{2}$ in accordance with Robinson's results. ${ }^{15}$ In the latter case, however, it seemed difficult for the solution to form the cholesteric structure when the polymer concentration was in a range between 12 and $24 \%(\mathrm{v} / \mathrm{v})$. For PELG in dioxane $Z_{0}{ }^{0}$ is proportional to $1 / C^{3.5}$. While $Z_{0}{ }^{0}$ increases with increasing temperature, no accurate figures for the temperature coefficient of $Z_{0}^{0}$, however, have been obtained owing to the marked hysterisis as Robinson ${ }^{15}$ pointed out except for PELG in dioxane; concn, $17.3 \%(\mathrm{v} / \mathrm{v})$.

Time Dependence of the Pitch in Magnetic Field The pitch of the uniform helix $Z_{0}^{0}$ undergoes time dependent change in a magnetic field as may be seen in Figure 2. It increases when the

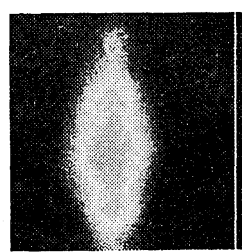

10

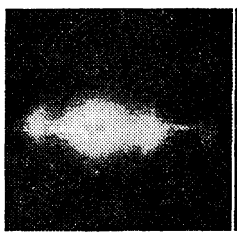

5

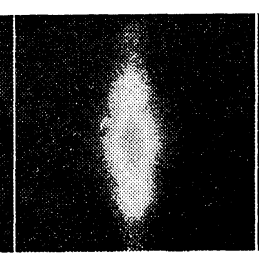

70

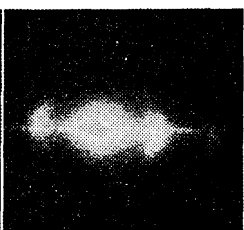

50

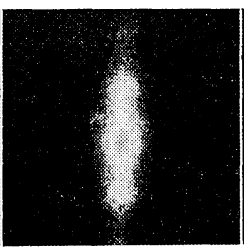

300

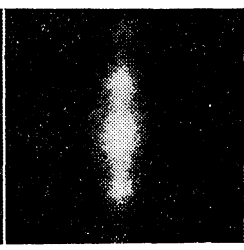

830

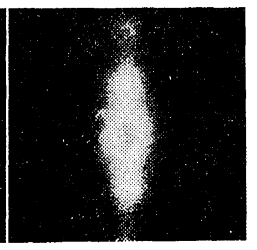

$2850 \mathrm{~min}$

Figure 2. Time dependence of the diffraction patterns in a magnetic field: PBLG in dioxane (16.0 vol \%), 9.6 kilogauss.

Direction of the magnetic field, parallel to the molecular layer (upper) or perpendicular to the molecular layers (lower). 


\section{E. IIzUKA}

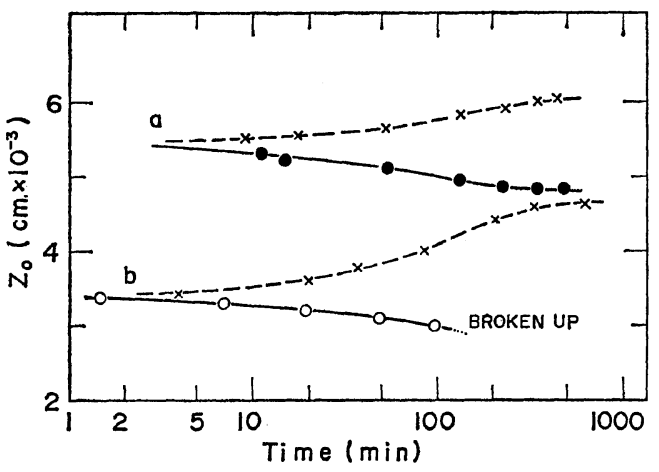

Figure 3. $Z_{0}{ }^{0}$ vs. time relationship: a, PBLG in $\mathrm{CH}_{2} \mathrm{Cl}_{2}$ (30 vol \%), 6 kilogauss; b, PBLG in dioxane $(11.0 \mathrm{vol} \%), 9.6$ kilogauss.

Direction of the magnetic field, perpendicular to the molecular layers (full lines) or parallel to the molecular layers (dotted lines). field direction is parallel to the molecular layers (the steady-state pitch will be described as $Z_{0}$, hereafter) and decreases only slightly when the field direction is perpendicular to the molecular layers (the steady-state pitch will be denoted as $Z_{0, \perp}$ hereafter). These relationships are shown in Figure 3. It has been known that the cholesteric structure is often broken up when the magnetic field is applied perpendicular to the molecular layers, whereas it is maintained when the magnetic field is applied parallel to the molecular layers.

Unlike the other systems observed, the change has been very rapid for dioxane solutions of PELG whose concentration is near to $17 \%(\mathrm{v} / \mathrm{v})$. Frenkel', and his coworkers ${ }^{20}$ have dealt with

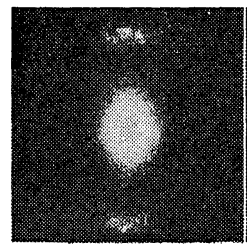

0

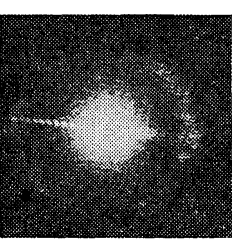

(a)

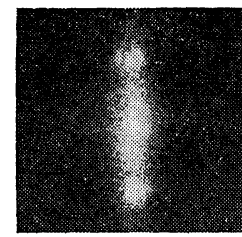

6

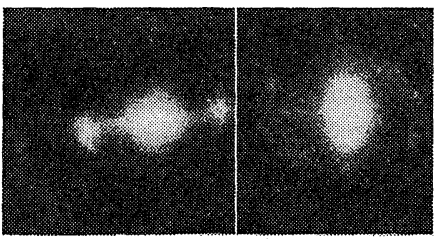

6

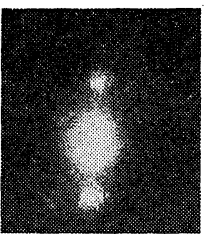

9.6
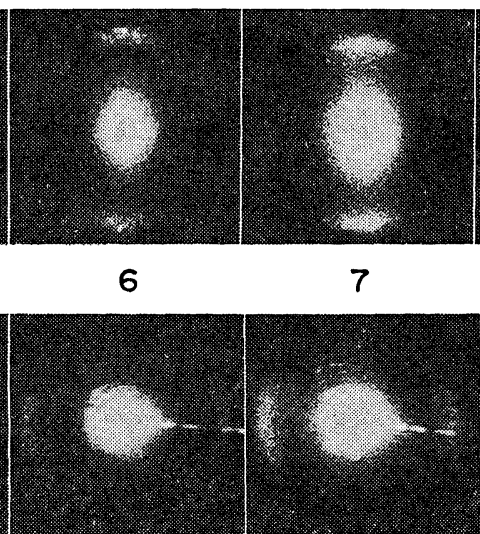

A

(b)

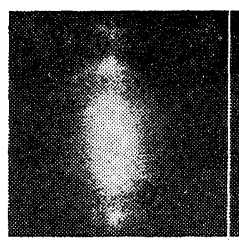

6

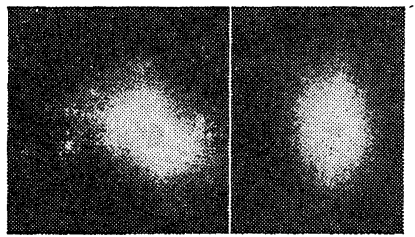

B

8

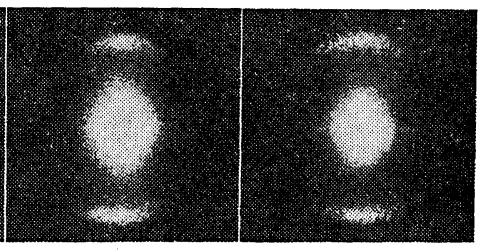

9 kgauss

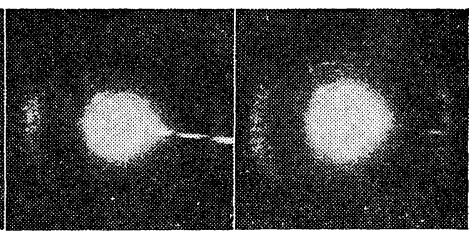

(c)

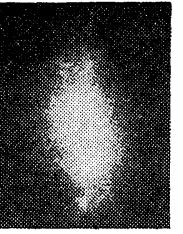

7

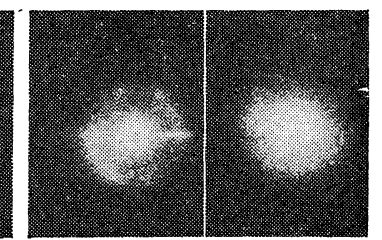

Figure 4. Dependence of the steady-state diffraction patterns upon field strength: A, PELG in dioxane $(14.0 \mathrm{vol} \%) ; \mathrm{B}(\mathrm{a}), \mathrm{PBLG}$ in dioxane $(11.0 \mathrm{vol} \%) ; \mathrm{B}(\mathrm{b}), \mathrm{PBLG}$ in $\mathrm{CH}_{2} \mathrm{Cl}_{2}$ (30 vol \%); B(c), PELG/GH $\mathrm{GH}_{2} \mathrm{Cl}_{2}(27 \mathrm{vol} \%)$.

Direction of the magnetic field, parallel (upper) or perpendicular (lower) to the molecular layers. 
The Effects of Magnetic Fields on the Structure of Cholesteric Liquid Crystals of Polypeptides

the phenomenology and thermodynamics of the specific ordered states in binary polymer-solvent systems, and have elucidated the transition of ordered states which occurs according to the changes in concentration and temperature. The fine structure of the cholesteric solution would be dependent on the system being observed. They also observed a sequence of liquid-crystalline states for PBLG in dichloroacetic acid at room temperature. A similar transition has been observed with a $\mathrm{CH}_{2} \mathrm{Br}_{2}$ solution of PBLG, that is, the cholesteric structure appears after about 2 weeks and this is substituted by another liquid-crystalline state after a while.

Effects of the Field Strength on the Pitch

Figure 4 shows that all the systems used are

(a)

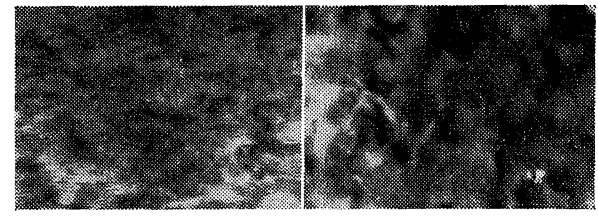

II

$$
\perp
$$

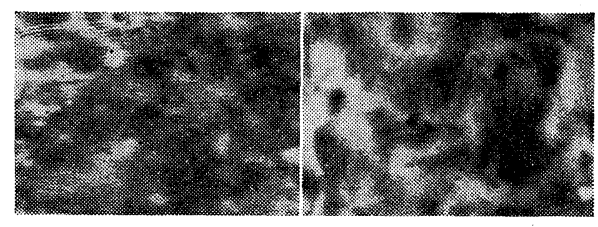

A

(a)

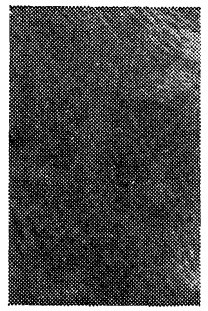

$30,40 x$ (b)

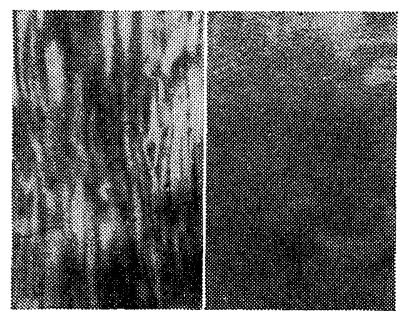

$11,40 x$
31 influenced by magnetic fields and that the influence is stronger when the field strength is raised. The change of the pitch is more explicitly shown in polarizing micrographs (see Figure 5).

The amount of increase in the pitch when the field direction is parallel to the molecular layers, $Z_{0, l l}-Z_{0}{ }^{0}$, is in proportion to $F^{4}$ where $F$ is the field strength as may be seen in Figure 6. According to Meyer, ${ }^{2} Z_{0, \|}$ is given by

$$
Z_{0, \|}=Z_{0}{ }^{0}\left[1+\frac{\left(\chi_{\mathrm{p}}-\chi_{\mathrm{t}}\right)^{2} F^{4}\left(Z_{0}^{0}\right)^{4}}{32(2 \pi)^{4} k_{22}^{2}}\right] \text {. }
$$

The experimental results are well expressed with this equation indicating that the curvatureelasticity theory is applicable to these viscoelastic

\section{(b)}

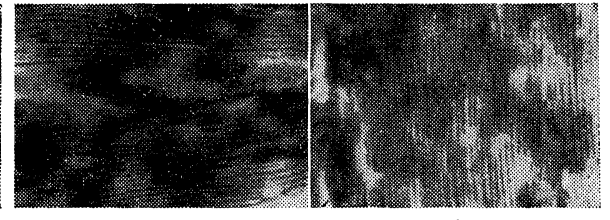

11

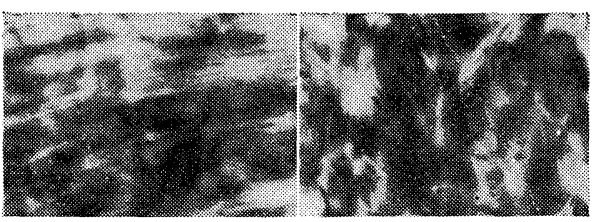

(c)

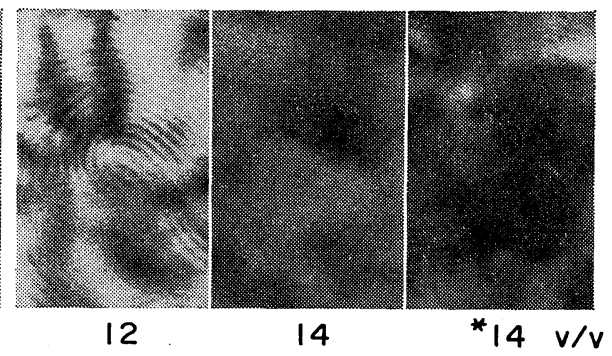

B

Figure 5. Polarizing micrographs of the cholesteric solutions and effects of magnetic field: $\mathrm{A}(\mathrm{a})$, PBLG in dioxane, $16.0 \mathrm{vol} \%, 22^{\circ} \mathrm{C} ; \mathrm{A}(\mathrm{b}), \mathrm{PBLG}$ in dioxane; $11.0 \mathrm{vol} \%, 32^{\circ} \mathrm{C} ; \mathrm{B}(\mathrm{a})$, PBLG in $\mathrm{CH}_{2} \mathrm{Cl}_{2} ; \mathrm{B}(\mathrm{b})$, PELG in $\mathrm{CH}_{2} \mathrm{Cl}_{2} ; \mathrm{B}(\mathrm{c})$, PELG in dioxane.

$A$, $(40 \times)$, upper, before the magnetic field was applied; lower, after the field was applied; B, $(100 \times)$, unless stated otherwise.

Numbers in the figure represent the polymer concentration in vol $\%$.

* shows the micrograph taken after a magnetic field (9.6 kilogauss) was applied parallel to the molecular layers. 
structures. Meyer, in deriving this equation, has assumed that the molecules are nonpolar with respect to the preferentially oriented axis, or if polar, are distributed with equal likelihood

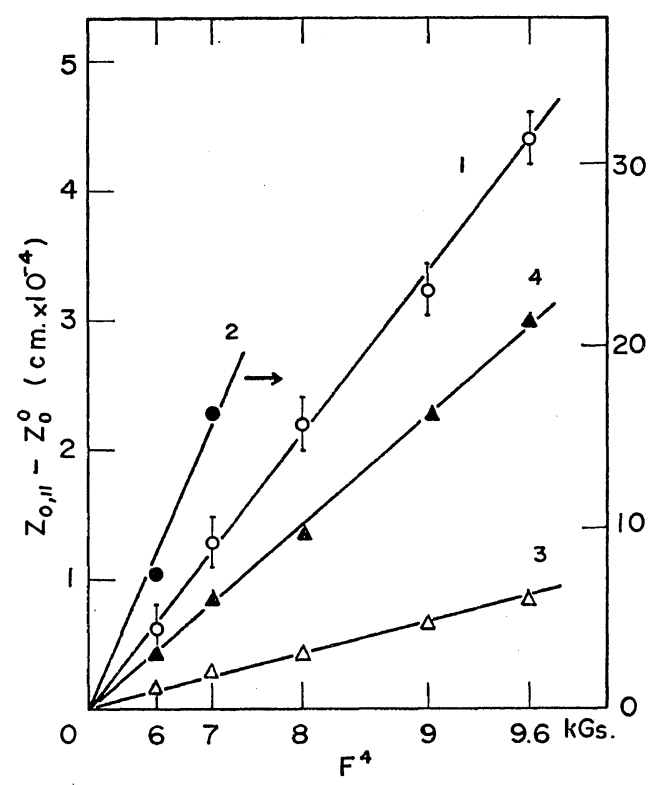

Figure 6. $Z_{0}, \|-Z_{0}^{0}$ vs. $F^{4}$ relationship: 1, PBLG in dioxane $(11.0 \mathrm{vol} \%)$; 2 , PBLG in $\mathrm{CH}_{2} \mathrm{Cl}_{2}$ (30 vol \%); 3, PELG in dioxane (14.0 vol \%); 4, PELG in $\mathrm{CH}_{2} \mathrm{Cl}_{2}(27 \mathrm{vol} \%)$. in both directions. As the polypeptide molecule is polar, the latter would be the case for the cholesteric structure. Table I summarizes the values of $k_{22} /\left(\chi_{\mathrm{p}}-\chi_{\mathrm{t}}\right)$ calculated from the slope of the lines in Figure 6, together with the values of the critical field strength $F_{c}$ calculated using the equation,

$$
F_{c}=\frac{2 \pi}{Z_{0}^{0}}\left[\frac{k_{22}}{\chi_{\mathrm{p}}-\chi_{\mathrm{t}}}\right]^{1 / 2}
$$

The same expression has been given by de Gennes $^{21}$ for the case of a magnetic field applied perpendicular to the optical axis of the structure. For this field, the pitch becomes infinite, as has been experimentally ascertained on amyloxy-azoxybenzene ${ }^{22}$ and a mixture of the derivatives of cholesterol. ${ }^{23}$

When the polymer concentration is increased, $k_{22} /\left(\chi_{p}-\chi_{t}\right)$ decreases and $F_{c}$ increases, probably due to increasing stability of the cholesteric structure. This represses the possible brownian movement of the polymer molecules and would increase an apparent magnetic anisotropy and make the pitch smaller as well. When the temperature is raised the brownian movement becomes intense, leading to a decrease in the magnetic anisotropy and the critical field strength. Experiments for determining the magnetic

Table I. Critical field strength and $k_{22} /\left(\chi_{\mathrm{p}}-\chi_{\mathrm{t}}\right)$

\begin{tabular}{|c|c|c|c|c|c|}
\hline Solute & Solvent & Concn & $\mathrm{T},{ }^{\circ} \mathrm{C}$ & $k_{22} /\left(\chi_{\mathrm{p}}-\chi_{\mathrm{t}}\right)$ & $F_{c}, \mathrm{kG}$ \\
\hline \multirow[t]{8}{*}{ PBLG } & Dioxane & $11 \%(\mathrm{v} / \mathrm{v})$ & 22.0 & $8.76 \pm 0.27$ & $6.50 \pm 0.10$ \\
\hline & & 16 & " & 5.9 & 7.0 \\
\hline & & 18 & 34.8 & 4.8 & 6.9 \\
\hline & & " & 41.0 & 5.0 & 6.6 \\
\hline & & " & 47.2 & 6.4 & 6.7 \\
\hline & & " & 53.0 & 4.8 & 5.8 \\
\hline & & 30 & 22.0 & 3.3 & 8.6 \\
\hline & $\mathrm{CH}_{2} \mathrm{Cl}_{2}$ & 30 & 21.4 & 12.2 & 4.1 \\
\hline \multirow[t]{11}{*}{ PELG } & Dioxane & 12 & 21.5 & 9.7 & 7.7 \\
\hline & & $" \prime$ & 38.6 & 24.2 & 6.4 \\
\hline & & 14 & 22.2 & 3.0 & 7.9 \\
\hline & & $" 1$ & 28.2 & 4.9 & 8.7 \\
\hline & & " & 33.3 & 6.1 & 8.0 \\
\hline & & " & 39.2 & 6.5 & 6.9 \\
\hline & & 17 & 22.0 & 1.2 & 12.5 \\
\hline & & $"$ & 39.7 & 1.6 & 8.9 \\
\hline & $\mathrm{CH}_{2} \mathrm{Cl}_{2}$ & 25 & 21.8 & 12.0 & 6.2 \\
\hline & & 27 & 22.0 & $6.22 \pm 0.12$ & $6.77 \pm 0.07$ \\
\hline & & 31 & 23.0 & 3.6 & 6.6 \\
\hline
\end{tabular}


The Effects of Magnetic Fields on the Structure of Cholesteric Liquid Crystals of Polypeptides

anisotropy of PBLG and PELG are now in progress and values of the order of $10^{-8}-10^{-9}$ $\mathrm{emu} / \mathrm{g}$ have been obtained, so far, in solution. ${ }^{24}$ From the result that the value of $k_{22} /\left(\chi_{\mathrm{p}}-\chi_{\mathrm{t}}\right)$ ranges from 1 to 10 , rough estimation for the value of $k_{22}$ will be made. The cholesteric structure is more stable in dioxane than in $\mathrm{CH}_{2} \mathrm{Cl}_{2}$ owing to the difference in the nature of solvent.

Comparison of the values of $F_{c}$ for the solutions of roughly the same concentration leads to the conclusion that with reference to a magnetic field the cholesteric structure of PELG is more stable than that of PBLG (refer to Table I), therefore it is safe to say that, the magnetic susceptibility (and the magnetic anisotropy as well) is smaller in the former. This difference would be due to the fact that the benzene groups having a diamagnetic effect are missing in PELG. In any case, it is still true that those polypeptides which do not have the benzene groups in their side chains also have an ability to make the magnetic orientation under appropriate conditions. As a matter of fact, when liquid crystals of PELG dissolved in $\mathrm{CH}_{2} \mathrm{Cl}_{2}$ are placed in a magnetic field of 14 kilogauss, the PMR signals of the solvent molecules split into doublets and the separation of the doublets changes according to the equation, $\left|h_{0}\left(3 \cos ^{2} \theta-1\right) / 2\right|$ where $h_{0}$ is the separation at $\theta=0$ and $\theta$ is the angle between the direction of orientation and that of the magnetic field. This suggests the orientation of PELG in the direction of the magnetic field (see ref 4) as shown in Figure 7.

It will be considered that when the liquidcrystalline solution undergoes a cholestericnematic transition in an external field, molecular clusters are formed in solution. The benzene rings of the PBLG molecule would be stacked among the neighboring molecules to help forming

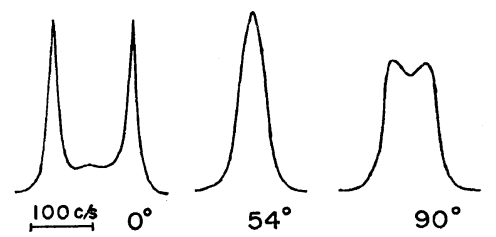

Figure 7. Dependence of the separation of the doublet upon the angle $\theta$ : PELG in $\mathrm{CH}_{2} \mathrm{Cl}_{2}-(20$ vol \%), $30^{\circ} \mathrm{C}$.

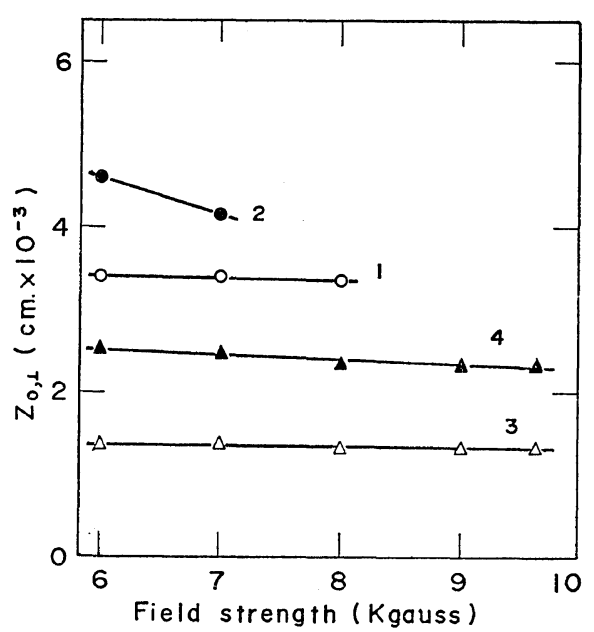

Figure 8. $Z_{0, \perp} v s$. field-strength relationship. See the footnote for Figure 6 .

a huge sheaflike aggregation of the molecules, which is considered to be more important for the ordering than the susceptibility for electric or magnetic field.

When the field direction is perpendicular to the molecular layers, the pitch, $Z_{0, \perp}$, decreases with increasing field strength though only slightly as may be seen in Figure 8. According to Meyer, ${ }^{2}$ when $k_{33}=k_{22}$ no perturbation takes place until $F_{c}$ is reached at which point complete break down of the cholesteric structure occurs and when $k_{33}<k_{22}, Z_{0, \perp}$ changes as follows:

$$
Z_{0, \perp}=\left(\frac{k_{33}}{k_{22}}\right)^{1 / 2} \cdot \frac{F_{c}}{F} Z_{0}^{0}
$$

in a range,

$$
\left(\frac{k_{33}}{k_{22}}\right)^{1 / 2} F_{c} \leqq F \leqq\left(\frac{k_{22}}{k_{33}}\right)^{1 / 2} F_{c} .
$$

The change in the pitch is not so marked as predicted for the latter case, but the cholesteric structure is still kept when $F_{c}$ is reached. This leads to the conclusion that $k_{33}$ is almost equal to, or slightly smaller than $k_{22}$.

\section{Effects of Electric Fields on the Cholesteric} Structure

The change of the pitch in electric fields is also time dependent. The $Z_{0, / l}-Z_{0}^{0}$ vs. $F^{4}$ relationship, however, does not follow the equation given by Meyer as may be seen in Figure 9. This discrepancy can be explained by considering the fact that permanent dipoles are 


\section{E. IIzUKA}

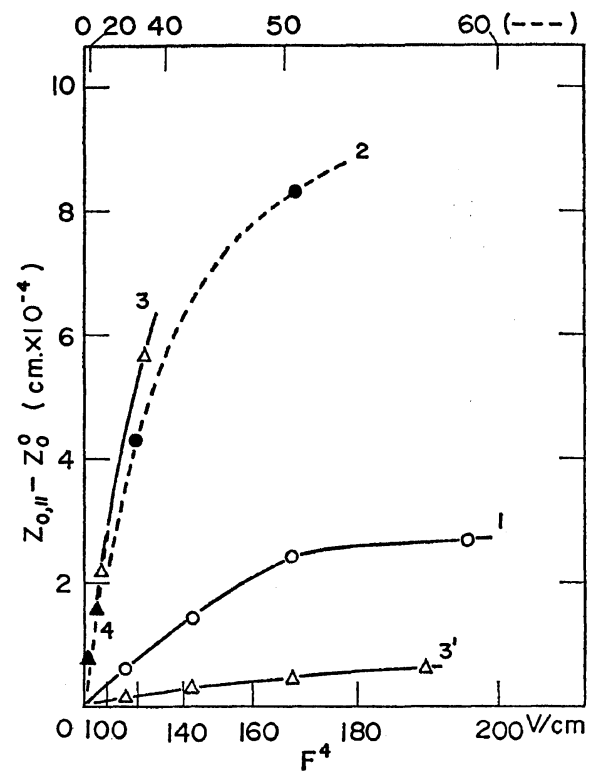

Figure 9. $Z_{0, / /}-Z_{0}^{0}$ vs. $F^{4}$ relationship for liquidcrystalline solutions in electric fields: 1, PBLG in dioxane (26 vol \%); 2, PBLG in $\mathrm{CH}_{2} \mathrm{Cl}_{2}$ (32 vol \%); 3 , PELG in dioxane $(12.0 \mathrm{vol} \%) ; 3^{\prime}$, PELG in dioxane (17.3 vol \%); 4, PELG in $\mathrm{CH}_{2} \mathrm{Cl}_{2}$ (31 vol \%).

involved in electric-field effects (in magneticfield effects induced dipoles are involved), whereas Meyer's calculation is based on the assumption that only induced dipoles are responsible for the field effects. Effects of electric fields on the cholesteric structure are still far from being analyzed at the present moment.

Acknowledgments. The author wishes to thank Prof. T. Hayakawa of this Faculty and Ajinomoto Co., for donating the polypeptide samples and Mitsubishi Steelworks Co., Ltd., for making the permanent magnet. His thanks are also due to Prof. A. Wada and Dr. T. Keira of The University of Tokyo for valuable discussion. Parts of this work was supported by the Grant-in-Aid for Individual Research (84032) from the Ministry of Education, Japan.

\section{REFERENCES}

1. C. Robinson, Trans. Faraday Soc., 52, 571 (1956).

2. R. B. Meyer, Appl. Phys. Letters, 12, 281 (1968).

3. F. C. Frank, Disc. Faraday Soc., 25, 19 (1958).

4. S. Sobajima, J. Phys. Soc. Japan, 23, 1070 (1967).

5. E. T. Samulski and A. V. Tobolsky, Macromolecules, 1, 555 (1968).

6. M. Panar and W. D. Phillips, J. Amer. Chem. Soc., 90, 3880 (1968).

7. Y. Go, S. Ejiri, and E. Fukada, Biochim. Biophys. Acta, 175, 454 (1969).

8. E. T. Samulski and A.V. Tobolsky, Mol. Cryst. Liq. Cryst., 7, 433 (1969).

9. E. T. Samulski and A. V. Tobolsky, Biopolymers, 10, 1013 (1971).

10. R. D. Orwoll and R. L. Vold, J. Amer. Chem. Soc., 93, 5335 (1971).

11. E. Iizuka and Y. Go, J. Phys. Soc. Japan, 31, 1205 (1971).

12. S. Yomosa, Abstracts, 9th Annual Meeting of Biophysical Society of Japan, Nagaya, Oct. 14, 1970, No. 1-C-8p.

13. M. Honda, H. Suzuki, and T. Kato, Rept. Progr. Polym. Phys. Japan, 14, 607 (1971).

14. E. Iizuka, Biochim. Biophys. Acta, 175, 457 (1969).

15. C. Robinson, J. C. Ward, and R. B. Beevers, Disc. Faraday Soc., 25, 29 (1958).

16. E. Iizuka, Biochim. Biophys. Acta, 243, 1 (1971).

17. E. Iizuka, T. Keira, and A. Wada, to be published.

18. S. Sobajima, private communication.

19. S. N. Stidham and R. S. Stein, J. Polym. Sci. Part. A-2. 4, 89 (1966).

20. S. Ya. Frenkel', L. G. Shaltyko, and G. K. Elyashevich, J. Polym. Sci. Part C., 30, 47 (1970).

21. P. G. de Gennes, Solid State Commun., 6, 163 (1968).

22. E. Sackmann, S. Meiboom, and L. C. Snyder, J. Amer. Chem. Soc., 89, 5981 (1967).

23. J. J. Wysocki, J. Adams, and W. Haas, Phys. Rev. Lett. 20, 1024 (1968).

24. K. Tohyama, N. Miyata, and E. Iizuka, unpublished results. 